\title{
Direct observation of microtubule-f-actin interaction in cell free lysates
}

\author{
Jenny R. Sider ${ }^{1,3}$, Craig A. Mandato ${ }^{1,3}$, Kari L. Weber ${ }^{1}$, Anna J. Zandy ${ }^{1}$, Dale Beach ${ }^{3,4}$, Rip J. Finst ${ }^{3,5}$, \\ Justin Skoble ${ }^{3,6}$ and William M. Bement ${ }^{1,2,3}$ \\ ${ }^{1}$ Department of Zoology and ${ }^{2}$ Program in Cellular and Molecular Biology, University of Wisconsin, Madison, WI, USA \\ ${ }^{3}$ Marine Biological Laboratory, Woods Hole, MA, USA \\ ${ }^{4}$ Department of Biology, University of North Carolina, Chapel Hill, NC, USA \\ ${ }^{5}$ Department of Cell Biology, Emory University School of Medicine, Atlanta, GA, USA \\ ${ }^{6}$ Department of Molecular and Cellular Biology, University of California, Berkeley, CA, USA \\ *Author for correspondence (e-mail: wmbement@vms2.macc.wisc.edu) \\ Accepted 22 March; published on WWW 26 May 1999
}

\section{SUMMARY}

Coordinated interplay of the microtubule and actin cytoskeletons has long been known to be crucial for many cellular processes including cell migration and cytokinesis. However, interactions between these two systems have been difficult to document by conventional approaches, for a variety of technical reasons. Here the distribution of $\mathbf{f}$ actin and microtubules were analyzed in the absence of fixation using Xenopus egg extracts as an in vitro source of microtubules and f-actin, demembranated Xenopus sperm to nucleate microtubule asters, fluorescent phalloidin as a probe for f-actin, and fluorescent tubulin as a probe for microtubules. F-actin consistently colocalized in a lengthwise manner with microtubules of asters subjected to extensive washing in flow chambers. F-actinmicrotubule association was heterogenous within a given aster, such that f-actin is most abundant toward the distal (plus) ends of microtubules, and microtubules heavily labeled with f-actin are found in close proximity to microtubules devoid of f-actin. However, this distribution changed over time, in that 5 minute asters had more f-actin in their interiors than did $\mathbf{1 5}$ minute asters. Microtubule association with f-actin was correlated with microtubule bending and kinking, while elimination of $\mathrm{f}$-actin resulted in straighter microtubules, indicating that the in vitro interaction between f-actin and microtubules is functionally significant. F-actin was also found to associate in a lengthwise fashion with microtubules in asters centrifuged through $30 \%$ sucrose, and microtubules alone (i.e. microtubules not seeded from demembranated sperm) centrifuged through sucrose, indicating that the association cannot be explained by flow-induced trapping and alignment of f-actin by aster microtubules. Further, cosedimentation analysis revealed that microtubule-f-actin association could be reconstituted from microtubules assembled from purified brain tubulin and f-actin assembled from purified muscle actin in the presence, but not the absence, of Xenopus oocyte microtubule binding proteins. The results provide direct evidence for an association between microtubules and f-actin in vitro, indicate that this interaction is mediated by one or more microtubule binding proteins, and suggest that this interaction may be responsible for the mutual regulation of the microtubule and actomyosin cytoskeletons observed in vivo.

Key words: Egg extract, Cytoskeleton, Cytokinesis, Xenopus

\section{INTRODUCTION}

One of the basic rules of eukaryotic cell motility is that the microtubule and actomyosin cytoskeletons can cooperate to execute complex changes in cell shape that result in cell movement and cell division. In simple terms, actomyosin provides the force for cell locomotion and cell fission in many cell types, while microtubules somehow direct that force. In the case of cell locomotion, actomyosin is required for cell protrusion, while microtubules are generally required to direct cell protrusion at the leading edge of the cell (reviewed by Vasiliev, 1991; Waterman-Storer and Salmon, 1999). In the case of cytokinesis, a contractile actomyosin apparatus pinches the cell in half, while microtubules are required for the assembly of the contractile apparatus between the separating chromosomes (reviewed by Fishkind and Wang, 1995; Oegema and Mitchison, 1997; Field et al., 1999).

A two system mechanism for cytokinesis was suggested by the work of Hiramoto (1956) more than forty years ago, and the relative roles of microtubules and actomyosin in cytokinesis have been established for more than two decades (Rappaport, 1961; Hiramoto, 1971; Schroeder, 1973; Hamaguchi, 1975; Fujiwara and Pollard, 1976; Mabuchi and Okuno, 1977). Likewise, observations of microtubule-f-actin cooperation in cell locomotion are longstanding (e.g. Vasiliev et al., 1970; Gail and Boone, 1971a,b; Goldman, 1971; Zigmond and Hirsch, 1972). However, in spite of intense interest, the nature of the interaction between microtubules and actomyosin has proven extraordinarily elusive, with a few specialized exceptions (see Gavin, 1997) for a variety of technical reasons. In particular, 
due to the polymeric nature of the two systems, potential microtubule-f-actin interactions are difficult to document by standard biochemical approaches (see Griffith and Pollard, 1982). Further, two significant technical difficulties attend simultaneous imaging of f-actin and microtubules by standard fluorescence microscopy-based approaches. First, many standard microtubule immunofluorescence protocols do not adequately preserve filamentous actin and vice versa (e.g. McBeath and Fujiwara, 1984; Vielkind and Swierenga, 1989 and references therein). Second, the high local concentration of $\mathrm{f}$-actin in cortical regions and in structures such as stress fibers tends to obscure visualization of less abundant pools of f-actin.

To overcome these difficulties, we have analyzed the relative distributions of microtubules and f-actin using Xenopus egg extracts. This system has previously been employed to study a variety of complex cellular processes including both microtubule (Verde et al., 1990; Belmont et al., 1990) and factin (Theriot et al., 1994; Ma et al., 1998) dynamics. The use of extracts offers two important advantages. First, addition of fluorescently modified tubulin to lysates permits visualization of microtubules in the absence of fixation (Belmont et al., 1990). Second, the lack of a cell boundary ensures that actin polymerization occurs in the absence of a background of intensely-staining, f-actin rich structures such as stress fibers and the cortex (Theriot et al., 1994). Using this system, we provide direct evidence of an interaction between microtubules and f-actin.

\section{MATERIALS AND METHODS}

\section{Preparation of egg extracts}

Interphase Xenopus egg extracts were prepared using a modification of the protocol of Newmeyer and Wilson (1991). Eggs were stripped from adult Xenopus females induced to ovulate as previously described (Murray, 1991), and washed repeatedly in $1 \times$ MMR $(10 \mathrm{mM}$ $\mathrm{NaCl}, 0.2 \mathrm{mM} \mathrm{KCl}, 0.1 \mathrm{mM} \mathrm{MgCl} 2,0.2 \mathrm{mM} \mathrm{CaCl}_{2}, 0.01 \mathrm{mM}$ EDTA and $0.5 \mathrm{mM}$ Hepes, $\mathrm{pH} 7.8$ ). Eggs were dejellied in $2 \%$ cysteine, rinsed in $0.25 \times \mathrm{MMR}$, rinsed twice in ELB buffer $(100 \mathrm{mM} \mathrm{KCl}, 0.1$ $\mathrm{mM} \mathrm{CaCl}_{2}, 1 \mathrm{mM} \mathrm{MgCl} 2,10 \mathrm{mM}$ K-Hepes, $\mathrm{pH}$ 7.7, $1 \mathrm{mM}$ DTT, and $250 \mathrm{mM}$ sucrose) and twice in ELB buffer $+10 \mu \mathrm{g} / \mathrm{ml}$ of the protease inhibitors pepstatin, leupeptin, chymostatin, aprotinin and $1 \mathrm{mM}$ Pefabloc (Boehringer-Mannheim, Indianapolis, IN). Eggs were then transferred to clear ultracentrifuge tubes, packed by a brief centrifugation in a clinical centrifuge, and the excess buffer was removed. The above protease inhibitors and cycloheximide were added to a final concentration (relative to packed egg volumes) of 10 $\mu \mathrm{g} / \mathrm{ml}$ and $100 \mu \mathrm{g} / \mathrm{ml}$, respectively. Eggs were crushed by spinning at $4^{\circ} \mathrm{C}$ for 20 minutes at $20,000 \mathrm{rpm}$ in an SW41 rotor, and the cytoplasmic layer was taken as previously described (Murray, 1991).

\section{Preparation of demembranated sperm}

Adult male Xenopus were killed by decapitation, their testes were removed, and then rinsed 3 times in ice-cold MBSH $(110 \mathrm{mM} \mathrm{NaCl}$, $2 \mathrm{mM} \mathrm{KCl}, 1 \mathrm{mM} \mathrm{MgSO} 4,0.5 \mathrm{mM} \mathrm{NaPO}_{4}, 2 \mathrm{mM} \mathrm{NaHCO} 3,15 \mathrm{mM}$ Tris-base, $\mathrm{pH}$ 7.6). Testes were then macerated in an ice-cold pyrex Petri plate in $4 \mathrm{ml}$ ice-cold HSPPP $(250 \mathrm{mM}$ sucrose, $15 \mathrm{mM}$ Hepes, $\mathrm{pH} 7.4,1 \mathrm{mM}$ EDTA, $0.5 \mathrm{mM}$ spermidine, $0.2 \mathrm{mM}$ spermine, 10 $\mu \mathrm{g} / \mathrm{ml}$ pepstatin, leupeptin, aprotinin, and chymostatin, $1 \mathrm{mM}$ Pefabloc) using the edge of four taped-together microscope slides. The macerated testes were then filtered through cheese cloth, pelleted in a clinical centrifuge $\left(4^{\circ} \mathrm{C}\right.$, setting 6), resuspended in HSPPP, repelleted, resuspended in $1 \mathrm{ml}$ room temperature $\mathrm{HSPPP}+500 \mu \mathrm{g} / \mathrm{ml}$ lysophosphatidylcholine (Boehringer-Mannheim), and incubated 5 minutes at room temperature. Sperm were then diluted in $10 \mathrm{ml}$ HSPPP $+0.3 \%$ BSA, pelleted, resuspended in HSPPP + BSA, pelleted again, resuspended at a final concentration of $\sim 1000 / \mu 1$ in HSPPP + $\mathrm{BSA}+30 \%$ glycerol, frozen in liquid nitrogen and stored at $-80^{\circ} \mathrm{C}$.

\section{Aster assembly and processing}

Flow chambers were constructed by inverting polylysine (Sigma; 1 $\mathrm{mg} / \mathrm{ml}$ )-coated coverslips over two pieces of parallel, double-stick tape adhered to microscope slides (Steve Block, Princeton University, personal communication). Flow chamber volumes were $\sim 10 \mu 1.50 \mu \mathrm{l}$ aliquots of demembranated sperm were thawed, $10 \mu \mathrm{l}$ were pipeted into each flow chamber, and allowed to adhere for 10 minutes. Chambers were then washed $5 \times$ with microtubule stabilization buffer (TSB; $80 \mathrm{mM}$ K-Pipes, pH 7.0, $5 \mathrm{mM} \mathrm{MgCl}_{2}, 1 \mathrm{mM}$ EGTA) containing $5 \mathrm{mg} / \mathrm{ml} \mathrm{BSA}$ to remove unbound sperm and to mask remaining areas coated with polylysine. Note that under these conditions only a small fraction of the sperm actually remain adhered to the flow chamber $(\sim 1-2 \%)$; the rest are washed through. Egg extracts were prepared for microtubule and f-actin analysis as follows: $50 \mu \mathrm{l}$ aliquots of extracts were thawed, and mixed with $2 \mu \mathrm{l}$ fluorescent tubulin $(10 \mathrm{mg} / \mathrm{ml}$; Cytoskeleton, Denver CO) and $2 \mu \mathrm{l}$ ATP regenerating system $(10 \mathrm{mM}$ creatine kinase, $10 \mathrm{mM}$ creatine phosphate, $1 \mathrm{mM}$ ATP; Leno and Laskey, 1991), and incubated on ice for two hours. The 2 hour incubation was not required for microtubule-f-actin interaction, however, in its absence, a number of small, ugly nuggets of clumped fluorescent tubulin were often visible in flow chambers.

After the ice incubation, $10 \mu \mathrm{l}$ of extract was flowed into chambers and incubated at room temperature for 15 minutes to allow microtubule and actin polymerization. The demembranated sperm are necessary to nucleate microtubule asters (Stearns and Kirschner, 1994) while the adherence of sperm to the chamber permitted subsequent washing of the samples. After extract incubation, chambers were washed $10 \times$ with $\mathrm{TSB}+5 \mathrm{mg} / \mathrm{ml} \mathrm{BSA}+20 \mu \mathrm{M}$ taxol (TSBT). Chambers were then incubated with TSBT containing $1 \mathrm{U} / \mathrm{ml}$ fluorescent phalloidin (either Oregon Green or Alexa 488 labelled; Molecular Probes, Eugene, OR) for 10 minutes and washed $5 \times$ with TSBT, and mounted in $80 \%$ glycerol containing $20 \mathrm{mM} \mathrm{N}$-propyl gallate to extract membranes, stabilize protein-protein interactions, and prevent photobleaching. We note that although no qualitative differences in results were observed when using fluorescein tubulin with Texas Red-phalloidin, rhodamine-tubulin yielded a superior microtubule signal. Consequently, all of the images and data presented were obtained using rhodamine tubulin as a probe for microtubules.

\section{Aster manipulation}

For cytochalasin treatments, cytochalasin D (Sigma) was diluted from a DMSO stock of $10 \mathrm{mg} / \mathrm{ml}$ into water to an intermediate stock concentration of $200 \mu \mathrm{g} / \mathrm{ml}$ and thence to a final concentration of 10 $\mathrm{mg} / \mathrm{ml}$ by addition of $1 \mu \mathrm{l}$ of the intermediate stock into $19 \mu \mathrm{l}$ of extract. Controls were subjected to addition of the same final concentration of DMSO alone. Following addition of the drug, samples were kept on ice for 2 hours. For extraction of asters with nonionic detergent, following the phalloidin staining step, chambers were washed $3 \times$ with TSB $+0.1 \%$ Triton $\mathrm{X}-100$ or, as a control, TSB alone. Both experimentals and controls were then washed again $5 \times$ with TSBT before mounting. For methanol extraction, experimentals and controls were processed as for Triton extraction, except that $100 \%$ methanol was used in place of TSB + Triton. For the methanol experiments, asters were extracted with methanol prior to staining with phalloidin, washed again with TSB to remove the methanol, and then stained with fluorescent phalloidin.

\section{Sedimentation of asters and microtubules assembled in extracts}

For analysis of asters and microtubules sedimented through sucrose, extracts were thawed and combined with rhodamine tubulin as above. 
To initiate aster formation and visualize asters, $1 \mu \mathrm{l}$ demembranated sperm was combined with $50 \mu$ l extract, warmed to room temperature for 15 minutes, and then rapidly diluted in $250 \mu \mathrm{TSB}+20 \mu \mathrm{M}$ taxol and $1 \mathrm{U} / \mathrm{ml}$ Alexa-488 phalloidin (Molecular Probes). Samples were then layered over $30 \%$ sucrose in TSB taxol, and immediately pelleted by centrifugation at $14,000 \mathrm{~g}$ for 5 minutes in a microfuge. Pellets were gently resuspended in mounting medium, and mounted for viewing by confocal fluorescence microscopy. To visualize microtubule-f-actin interactions in the absence of asters, extracts were prepared in the same way, but demembranated sperm were omitted. After 15 minutes at room temperature, extracts were diluted as above, and centrifuged through $30 \%$ sucrose at 20 psi for 20 minutes in an airfuge. Pellets were resuspended in mounting medium and mounted for viewing by confocal fluorescence microscopy.

\section{Confocal fluorescence analysis and image processing}

Samples were analyzed using a Bio-Rad 1024 confocal fluorescence microscope at the Keck Center for Neuroscience, University of Wisconsin. For all quantitative comparisons, gain and iris settings were held constant within a given experiment. All images were obtained using a $\times 60$ 1.4 NA objective lens; higher magnification images were obtained using the zoom function. All quantitative comparisons were done on images obtained without the zoom. Images were processed and analyzed using Adobe Photoshop. For quantification of fluorescence intensities within individual asters, raw images (i.e. unprocessed images) of asters were outlined either completely or as separate regions (see Results) and both the total size of the outlined region and the mean signal of fluorescent tubulin and fluorescent phalloidin were determined. Total signal was determined by multiplying the mean signals by the size of the aster or aster region. Background signal was determined by measuring mean signal intensities in regions adjacent to asters, and then multiplying the means by the total aster size. Final fluorescent signals were determined by subtracting the background signal from the total signal. All comparisons were conducted using control and experimental manipulations from the same experiment. Statistical analyses were performed using Microsoft Excel.

\section{Preparation of oocyte microtubule binding proteins}

Crude oocyte microtubule binding protein (MBPs) fractions were obtained from Xenopus oocytes prepared by collagenase treatment as previously described (Bement and Capco, 1989). After collagenase treatment, oocytes were washed extensively, and allowed to recover at $18^{\circ} \mathrm{C}$ overnight. $10 \mathrm{ml}$ of oocytes were then incubated for 1 hour at room temperature in $20 \mu \mathrm{M}$ latrunculin B (Calbiochem, La Jolla, CA) to disassemble actin filaments, and then subjected to a 1 hour cold shock on ice to disassemble microtubules. Oocytes were homogenized with 10 strokes in a Potter-Elvehjem homogenizer in an equal volume of ice-cold TSB containing $0.5 \%$ Triton X-100, $1 \mathrm{mM}$ DTT, $10 \mu \mathrm{g} / \mathrm{ml}$ pepstatin, $10 \mu \mathrm{g} / \mathrm{ml}$ leupeptin, $10 \mu \mathrm{g} / \mathrm{ml}$ chymostatin, $10 \mu \mathrm{g} / \mathrm{ml}$ aprotinin, and $1 \mathrm{mM}$ Pefabloc. Yolk was pelleted by centrifugation at $15,000 \mathrm{~g}$ for 20 minutes. The supernatant was then subjected to a $100,000 \mathrm{~g}$ clearing centrifugation at $4^{\circ} \mathrm{C}$ for 1 hour. Supernatants were removed, warmed to room temperature, and taxol was added stepwise to a final concentration of $20 \mu \mathrm{M}$. Microtubule polymerization was allowed to proceed for 1 hour at room temperature and then microtubules and associated MBPs were pelleted by centrifugation though a $30 \%$ sucrose cushion at $100,000 \mathrm{~g}$ for 30 minutes (see Fig. 8A, lane 1). Pellets, containing microtubules and associated MBPs, were resuspended in TSB-taxol, and then mixed with TSB $+\mathrm{NaCl}$ and ATP at a final (after mixing) concentration of $1 \mathrm{M}$ and $10 \mathrm{mM}$, respectively (to extract MBPs) and then centrifuged through sucrose again to remove microtubules. Supernatants, containing the MBPs (see Fig. 8A, lane 2), were mixed with $30 \%$ sucrose, snap frozen in liquid $\mathrm{N} 2$ in $50 \mu \mathrm{l}$ aliquots, and stored at $-80^{\circ} \mathrm{C}$ until further use. Prior to use, MBP fractions were thawed, desalted using Centricon-10 columns, and then centrifuged at 100,000 $\mathrm{g}$ for 1 hour to remove particulates. Although this protocol does not entail classic microtubule 'cycling', it is similar to that used in the isolation of microtubule binding proteins such as kinesin (Vale et al., 1985) and CLIP-170 (Rickard and Kreis, 1990).

\section{Reconstitution of microtubule-f-actin interactions}

Purified muscle actin (Cytoskeleton) was thawed, diluted in low salt buffer on ice, and centrifuged to remove aggregates. Polymerization was initiated by addition of $\mathrm{KCl}$ to a final concentration of $50 \mathrm{mM}$ and warming to room temperature for 1 hour. Filaments were then stabilized with phalloidin $(10 \mu \mathrm{M})$, pelleted at $100,000 \mathrm{~g}$ for 1 hour, resuspended, and stored on ice until later use. Purified brain tubulin (Cytoskeleton) was thawed, polymerized by the addition of taxol, and then pelleted by centrifugation at $100,000 \mathrm{~g}$. Microtubules were resuspended in TSB-taxol, and stored on ice until later use.

Microtubules $(25 \mu \mathrm{l}$ at $80 \mu \mathrm{g} / \mathrm{ml})$ and f-actin $(25 \mu \mathrm{l}$ at $80 \mu \mathrm{g} / \mathrm{ml})$ were combined with each other or with $25 \mu$ l polymerization buffer and then coincubated in the presence or absence of $10 \mu \mathrm{l}$ oocyte MBPs $(50 \mu \mathrm{g} / \mathrm{ml})$ at room temperature for 30 minutes, and then centrifuged at $6,000 \mathrm{~g}$ for 5 minutes. In separate experiments, microtubules and f-actin were separately preloaded with MBPs by incubation for 30 minutes at room temperature followed by centrifugation through $30 \%$ sucrose at $66,000 \mathrm{~g}$ for 5 minutes. Pellets were resuspended and then combined and centrifuged as above. Pellets and supernatants were boiled in SDS-sample buffer and loaded stoichiometrically on $10 \%$ SDS-polyacrylamide gels. After electrophoresis, gels were silver stained or Coomassie stained. Results shown are representative of five different experiments.

\section{RESULTS}

\section{F-actin colocalizes with astral microtubules in vitro}

Microtubule aster formation was initiated and visualized by adding 'interphase' (Murray, 1991) egg extracts with rhodamine-labeled tubulin to flow chambers containing demembranated sperm (Stearns and Kirschner, 1994) adhered to polylysine-coated coverslips. Following addition of lysates, chambers were incubated for 15 minutes to allow microtubule and f-actin polymerization and then washed repeatedly with microtubule stabilization buffer containing taxol to stabilize microtubules and to remove the lysate as well as anything that was not bound to astral microtubules. Chambers were stained with Oregon Green-labeled phalloidin to probe for f-actin, washed again, and then mounted in glycerol to extract membranes and stabilize protein-protein interactions. Samples were then analyzed by laser scanning confocal microscopy.

In more than 50 experiments using eight different egg extract preparations and five different sperm preparations, f-actin consistently colocalized with astral microtubules. Fig. 1A shows low magnification examples of asters from five different experiments. The absolute amount of f-actin associated with asters varied from experiment to experiment, however, the spatial distribution of f-actin within flow chambers mirrored that of asters (Fig. 1A). This point was confirmed by comparing the relative fluorescence intensity of the phalloidin and tubulin signals in astral vs nonastral regions (see Materials and Methods). In three independent experiments, the spatial distribution of the phalloidin signal was correlated with the distribution of the tubulin signal (correlation coefficients $=0.8$, 0.8 , and $0.79 ; P<0.05$ for all three experiments).

Because samples were mounted in $80 \%$ glycerol prior to viewing, it is unlikely that the observed interaction of f-actin 

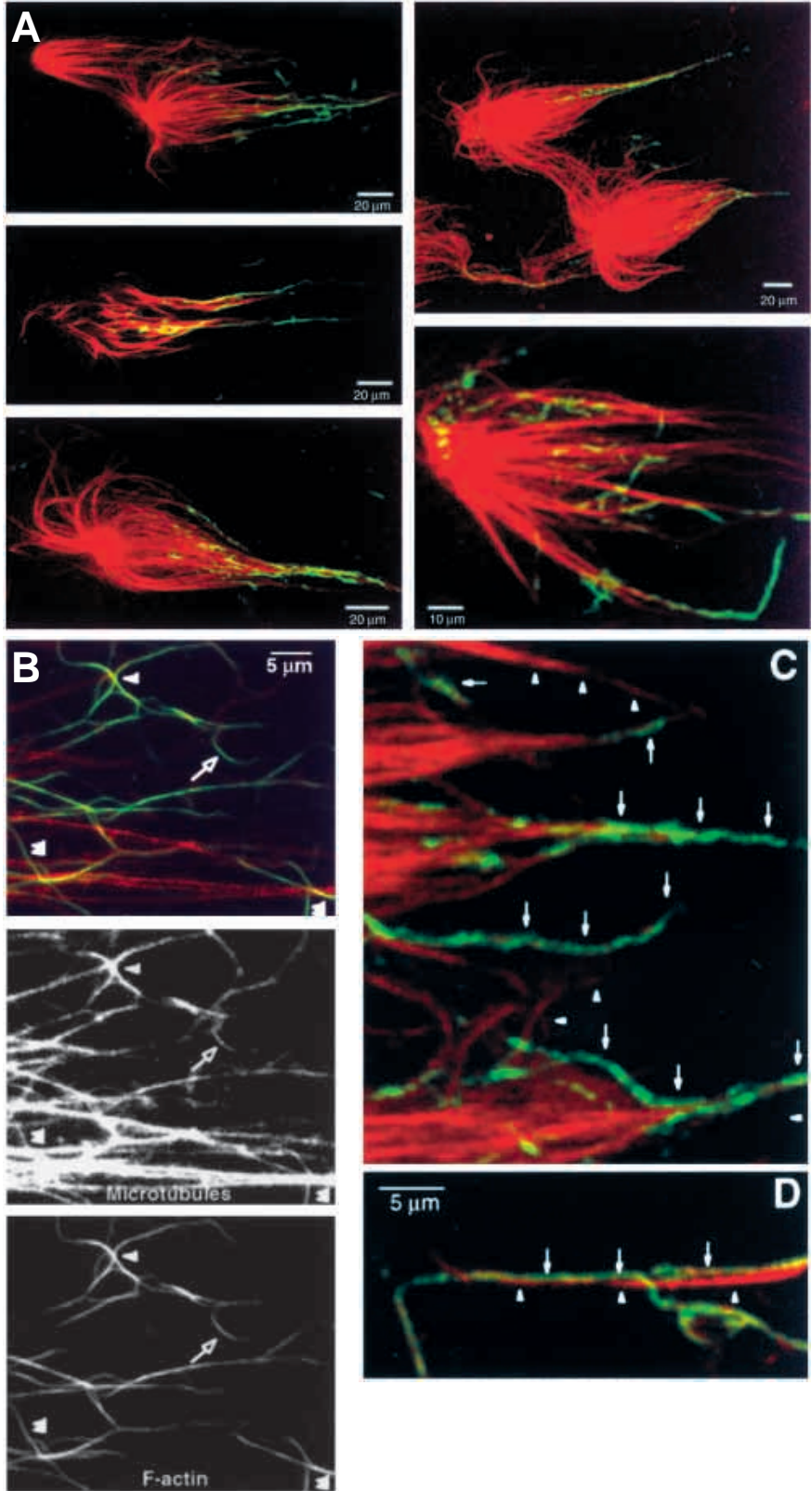

Fig. 1. F-actin colocalizes with microtubule asters in vitro. (A) Low magnification confocal fluorescence micrographs showing codistribution of astral microtubules (red or yellow) and f-actin (green or yellow). (B) High magnification confocal micrographs showing that f-actin runs parallel to microtubules. Top panel: dual label showing microtubules (red or yellow) and f-actin (green or yellow). Note that in some areas, the intensity of the green signal obscures any underlying red signal. Bottom panels: image is split into individual red and green channels showing that even areas of $\mathrm{f}$ actin staining that run perpendicular to most of the rest of the microtubules (double arrowheads) or which have no apparent associated red signal in the double label image (arrow) are nevertheless associated with microtubules. Single arrowhead indicates an area where several microtubules with associated f-actin come together. (C) High magnification image showing tips of adjacent microtubules (red) with heavy f-actin (green) labeling (arrows) or no f-actin labeling (arrowheads). (D) High magnification image showing two microtubules in immediate contact with heavy factin labeling (arrows) or no f-actin labeling (arrowheads). with asters is mediated by membrane-dependent linkages. To further assess the role of membranes, asters were washed with $0.1 \%$ Triton $\mathrm{X}-100$, a manipulation previously shown to extract membranes in Xenopus egg extracts (Allan and Vale, 1991). Triton washing prior to mounting and viewing did not significantly reduce the amount of microtubule-associated $\mathrm{f}$ actin $(81 \pm 26 \%$ of controls, mean \pm s.e.m., $P>0.05 ; n=2)$. In contrast, methanol extraction, which is frequently employed for immunofluorescence staining of microtubules (e.g. Vielkind and Swierenga, 1989), sharply reduced the amount of microtubule-associated, fluorescent-phalloidin signal $(11 \pm 2 \%$ of controls, mean \pm s.e.m., $P<0.05 ; n=2$ ), presumably due to methanol-dependent inhibition of phalloidin-f-actin binding (e.g. Howard and Oresajo, 1985).

While low magnification images (Fig. 1A) showed that factin codistributes with asters, it was not clear whether f-actin is simply intertwined with astral microtubules. However, high magnification views revealed that the majority of f-actin associates with microtubules in a lengthwise fashion (Fig. 1B). This was especially apparent when double-label images were split into the individual channels. Even f-actin that did not run parallel to the long axis of the aster (Fig. 1B, double arrowheads), or which appeared to have no association with microtubules in the dual fluorescence image (Fig. 1B, arrows), was clearly seen to be associated with microtubules. Such images also showed that continuous f-actin staining was often associated with converged, parallel microtubules, as if f-actin linked microtubules in a sideways manner (Fig. 1B, arrowhead).

\section{F-actin-microtubule association is heterogenous}

In a given field of view, the spatial distribution of f-actin was tightly correlated with the spatial distribution of asters (Fig. 1A), suggesting that $\mathrm{f}$-actin that does not bind microtubules is washed out of the flow chamber. However, within a given aster, the association of f-actin with microtubules was nonuniform. That is, individual microtubules differed significantly in the amount of associated f-actin. Microtubules with high levels of associated f-actin (Fig. 1C, arrows) were often found in close proximity to microtubules with little or no associated f-actin (Fig. 1C, arrowheads). In extreme cases, microtubules with heavy f-actin labeling (Fig. 1D, arrows) were found in immediate proximity to microtubules devoid of f-actin (Fig. 1D, arrowheads).

Casual inspection of asters suggested that f-actin labeling was generally most intense at or near the distal ends of microtubules (Figs 1A,C,D, 2A). To test this point directly, the ratio of phalloidin:tubulin signal was quantified within regions of asters polarized by the washing process at increasing distances from the aster base (Fig. 2A). Phalloidin:tubulin signal was significantly higher in regions distal to the aster base (Fig. 2B).

To address the possibility that the association of f-actin with microtubules might be dynamic, the relative distribution of $\mathrm{f}$ actin on microtubules was compared between asters allowed to nucleate microtubules for different lengths of time. That is, samples were prepared as described above after either a 5 minute or 15 minute incubation. As described above, late (15 minute) asters had most of their associated f-actin at the distal ends of microtubules (Fig. 3). In contrast, early (5 minute) asters consistently showed significantly less f-actin toward the 

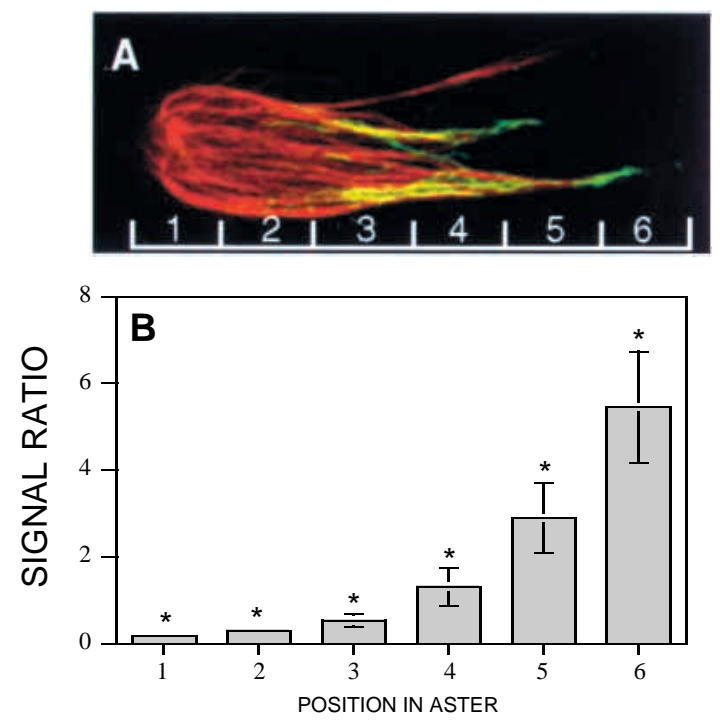

Fig. 2. F-actin preferentially associates with the distal ends of microtubules. (A) Confocal micrograph of a polarized aster showing f-actin (green or yellow) concentrated toward the distal ends of the microtubules (red or yellow) and the division of the aster into six regions of equal length. (B) Histogram showing mean factin:microtubule signal ratios in different regions of polarized asters. Results represent analysis of 54 asters from three independent experiments, \pm s.e.m. Asterisks indicate significant differences relative to region $1(P<0.05)$.

distal ends of microtubules than did late asters (Fig. 3), suggesting that f-actin may redistribute toward the aster periphery over time, although we cannot rule out that the apparent change in distribution results from differences in probe accessibility at the different time points.

While the majority of f-actin was localized in a length-wise manner toward the distal ends of microtubules, a second pattern of f-actin-aster colocalization was occasionally observed. Specifically, semi-circular arcs or 'comets' of f-actin were sometimes observed at the base of asters, in the area of the microtubule organizing center (Fig. 4). Such structures were found in large, well-spread asters that extended from a large, spherical microtubule organizing center. In contrast to factin associated with the distal ends of microtubules, f-actin

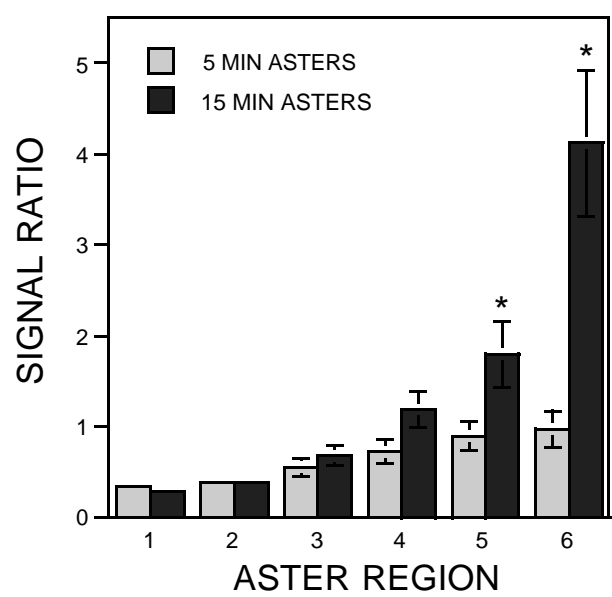

Fig. 3. F-actin distribution with respect to position in asters changes over time. A histogram showing mean f-actin:microtubule signal in different regions of early (5 minute) and late (15 minute) asters, quantified as in Fig. 4. Results represent analysis of 73 asters from three independent experiments \pm s.e.m. Asterisks indicate significant differences between 5 minute and 15 minute asters $(P<0.05)$.

arcs appeared to be positioned in areas that had relatively few microtubules. That is, arcs were concentrated inside the area from which microtubules appeared to extend (Fig. 4). These arcs were not contained within the decondensing sperm nuclei, as judged by inspection of samples using phase microscopy (not shown).

\section{Association with f-actin results in microtubule bending}

Microtubules at the edges of asters were occasionally observed to have sharp bends or kinks (Fig. 5A). Sharply bent microtubules were associated with heavy f-actin staining (Fig. 5A), suggesting that interaction with f-actin promotes microtubule bending. To explore this possibility further, asters were assembled in extracts lacking or containing cytochalasin $\mathrm{D}$ (CD), an agent which inhibits actin polymerization (Cooper, 1986). CD treatment eliminated staining of astral microtubules with f-actin, except for isolated punctae (not shown). Thus, the phalloidin probe did not nonspecifically bind to either the astral
Fig. 4. F-actin arcs are found in the area of the microtubule organizing center in large, well-spread asters. Left panel: low magnification, dual label image of a large, well-spread aster with a spherical microtubule organizing. F-actin (green or yellow) is observed at both the tips of the microtubules and within the microtubule organizing center. Right panels: enlarged view of the microtubule organizing center, split into the individual channels. The factin is in the shape of whorls or comets and is found in a region with very few microtubules.
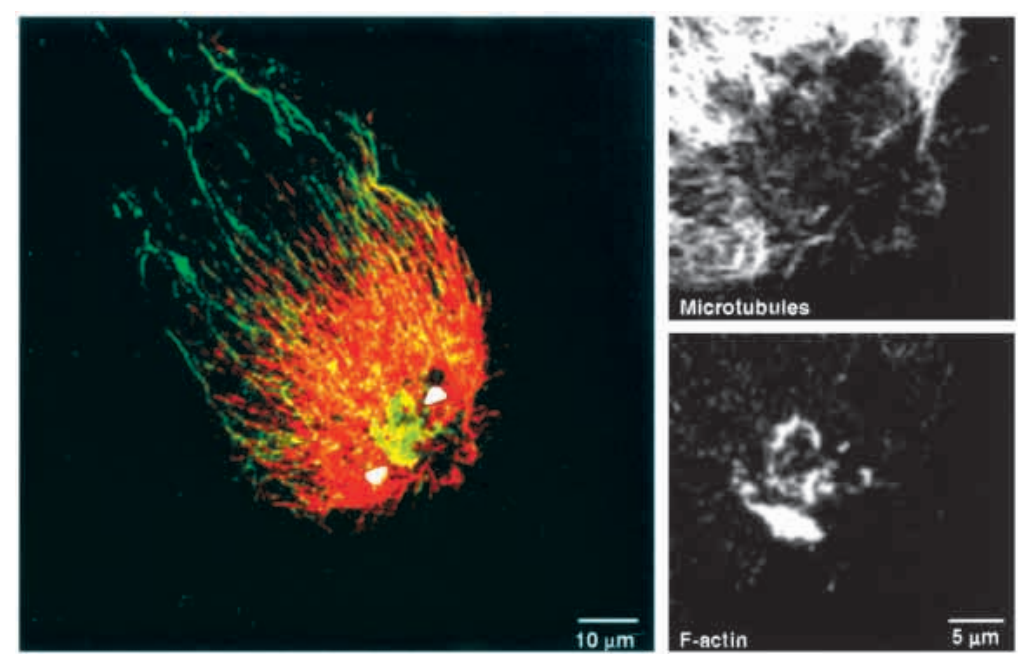
Fig. 5. Association with f-actin results in microtubule kinking. (A) High magnification views showing sharply bent microtubules (arrowheads) associated with heavy f-actin staining.

(B) Microtubules in asters formed in the presence (control) or absence (cytochalasin D) of f-actin.

Microtubules in control asters appear less smooth than in asters formed in the absence of f-actin. (C,D) Quantification of $f$-actin induced kinking of microtubules. Asters formed in the presence of f-actin pack less tightly than those formed in its presence and have significantly more sharply bent microtubules. Results represent analysis of 60 asters from the mean \pm s.e.m. from three independent experiments Asterisk indicates difference with $P<0.05$.

microtubules themselves or non-actin structures associated with them. More importantly, astral microtubules formed in the presence of $\mathrm{CD}$ appeared straighter and smoother than those formed in its absence (Fig. 5B). This impression was confirmed by analysis of two parameters. First, mean tubulin fluorescence intensity vs total aster size was determined in images of isolated, polarized asters obtained in the presence and absence of $\mathrm{CD}$, based on the rationale that asters composed of straighter microtubules should pack tighter during washing and therefore have a higher mean signal:size ratio than asters composed of bent microtubules. The mean signal:size ratio was significantly higher in asters formed in the presence of CD than those formed in its absence (Fig. 5C). Second, the number of sharply bent $\left(90^{\circ}\right.$ or greater within $2 \mu \mathrm{m}$ microtubule length) microtubules was quantified for asters grown in the presence and absence of $\mathrm{CD}$. The inclusion of $\mathrm{CD}$ reduced the number of sharply bent microtubules by more than $80 \%$ (Fig. 5D). Although the number of bent microtubules per aster calculated for control samples was small $(\sim 1)$, it is likely that this is an underestimate, in that bent or broken microtubules in the interior of the aster could not be quantified and since most broken microtubules would presumably be removed during washing.

\section{F-actin-microtubule association in asters and microtubules centrifuged through sucrose}

To determine whether the observed f-actin-microtubule association was simply an artifact of the flow chamber approach, f-actin-microtubule association was assessed in asters sedimented through $30 \%$ sucrose, a standard approach to identify microtubule-associated proteins and structures. Asters were generated by direct addition of demembranated sperm to extracts containing rhodamine-tubulin. After a 15 minute incubation, extracts were diluted in TSB containing taxol to stabilize microtubules and Alexa-488 phalloidin to stabilize and label f-actin and then spun through a $30 \%$ sucrose cushion. Pellets were resuspended in TSB-taxol and analyzed by confocal microscopy. As observed in asters seeded and processed in flow chambers, f-actin associated with astral microtubules in an apparently lengthwise fashion, as judged by the outward radiation of f-actin from the aster centers (Fig. 6A). The heaviest f-actin labeling was observed at the distal ends of asters, consistent with the flow chamber observations. Further, the correlation between the distribution of f-actin and microtubules observed in flow chambers was also observed in asters sedimented through sucrose (results represent analysis of 38 asters from two experiments, correlation coefficients $=0.82,0.81 ; P<0.05$ for both experiments).

To determine whether f-actin-microtubule association required aster formation, or could occur with smaller microtubule arrays, extracts containing rhodamine tubulin were warmed to room temperature for 15 minutes without addition of demembranated sperm, diluted in TSB containing taxol and Alexa-488 phalloidin as above, and sedimented through sucrose. Pellets were resuspended in TSB-taxol and analyzed by confocal microscopy. Such pellets contained factin associated with microtubules (Fig. 7), indicating that factin microtubule association does not depend on formation of large microtubule arrays. As above, microtubule-associated factin in these preparations ran parallel to microtubules, although in some cases the region of interaction was relatively short (Fig. 7A, compare interactions indicated by arrows and arrow heads; Fig. 7B). Sedimentation through sucrose in the absence of asters would be expected to preferentially pellet 
Fig. 6. F-actin associates with microtubules in asters sedimented through $30 \%$ sucrose. Double label (first panel) and split label (second and third panels) confocal images showing distribution of microtubules (red or yellow in first panel) and f-actin (green or yellow in first panel) in asters assembled in extracts and then sedimented through sucrose by centrifugation. The factin codistributes with the astral microtubules in a lengthwise fashion, rather than lying perpendicular to the long axis of the aster microtubules. Note also that the f-actin labeling appears to be heaviest at the periphery of the asters.
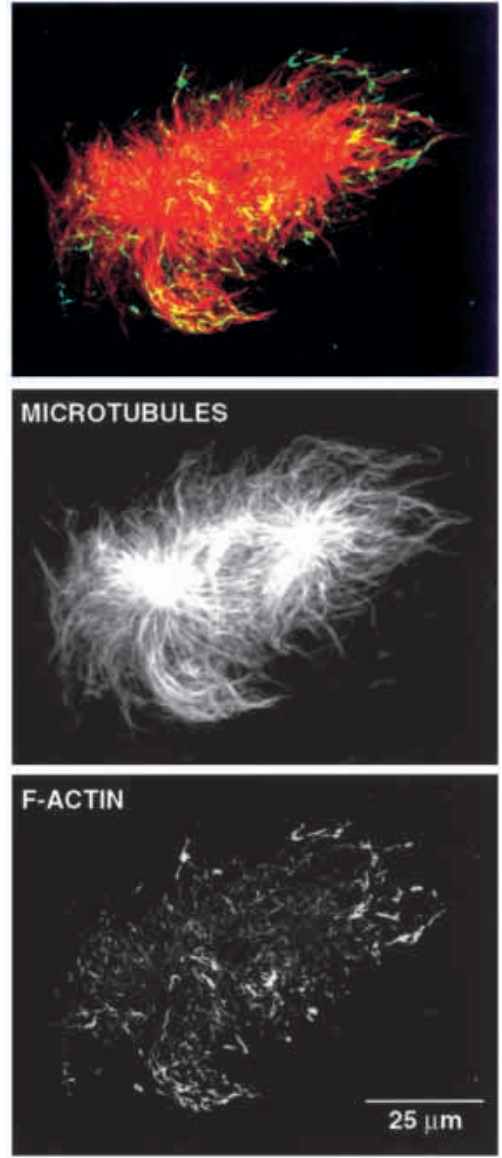

Fig. 7. F-actin associates with microtubules assembled in the absence of microtubule organizing centers and sedimented through sucrose. Confocal micrographs of microtubules assembled in the absence of demembranated sperm and then spun through $30 \%$ sucrose; F-actin is green or yellow; microtubules are red or yellow. (A) F-actin associates with microtubules in a lengthwise fashion for long (arrows) or short (arrowhead) distances. Asterisk indicates what appears to be a single microtubule with $\mathrm{f}$-actin running along its length. Note that f-actin appears to crosslink different microtubule bundles (middle of frame). (B) Another example of $\mathrm{f}$ actin associated in a lengthwise fashion with a
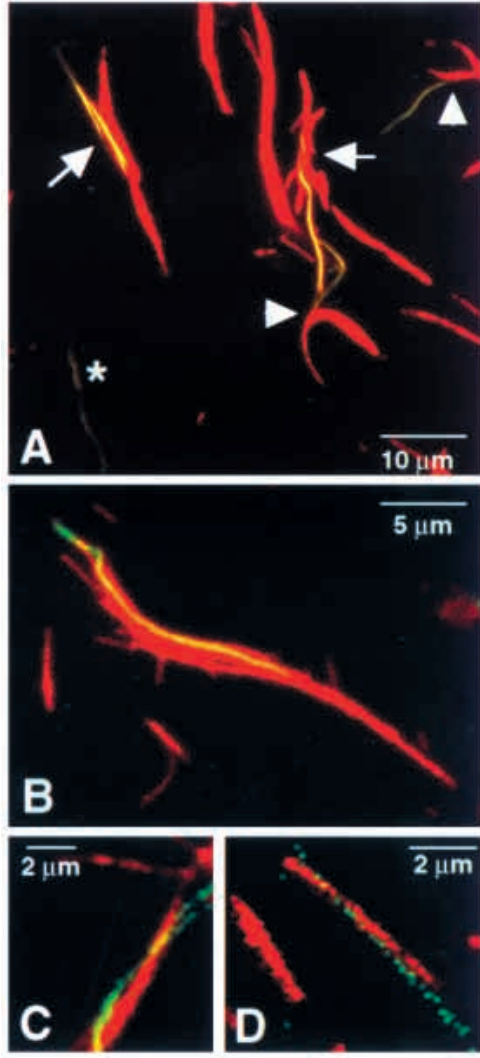

bundle of microtubules. (C) High magnification view showing a relatively small $\mathrm{f}$-actin bundle associated in a lengthwise fashion with a relatively small microtubule bundle. (D) High magnification view showing a very lightly fluorescent f-actin filament or cable associated with what may be an individual microtubule in a lengthwise fashion.

larger microtubule-f-actin structures and, indeed, most of the interactions observed were between what appeared to be bundles composed of several microtubules and f-actin cables (Fig. 7A, arrows; Fig. 7B). However, interactions between smaller/less intensely-staining structures were also observed (Fig. 7A, asterisk; Fig. 7C,D). Thus, the observed association of f-actin with microtubules is dependent on neither the flow chamber approach nor the presence of large microtubule structures such as asters.

\section{MBP-dependent reconstitution of f-actin- microtubule association}

Pure microtubules do not associate with pure actin filaments (e.g. Griffith and Pollard, 1978), so it seemed likely that the observed interaction of $\mathrm{f}$-actin with microtubules was mediated by one or more microtubule binding proteins (MBPs). Extraction with high salt concentrations is a standard approach for removing MBPs from taxol-stabilized microtubules. However, after trying a variety of different protocols using all of the above assays, we found that while high salt (0.5-1 M $\mathrm{KCl}$ or $\mathrm{NaCl}$ ) extraction disrupted f-actin microtubule association, it also destroyed asters (not shown), presumably by disrupting interactions between astral microtubules and the MTOC. In addition, while high salt extraction stripped f-actin associated with microtubules assembled in the absence of MTOCs, it also completely eliminated f-actin bundles (not shown), preventing meaningful comparison to controls samples, which contain many bundles (Fig. 7).
Therefore, to address the potential role of MBPs as f-actinmicrotubule linkers, microtubules assembled from porcine brain tubulin were mixed with f-actin assembled from muscle actin in the presence and absence of a MBP fraction obtained from Xenopus oocytes (Fig. 8A; see also Materials and Methods). F-actin, microtubules, and MBPs were subjected to low-speed $(5,000 \mathrm{~g})$ centrifugation alone, or after coincubation, and then analyzed by SDS-PAGE. The combination of MBPs with f-actin or MBPs with microtubules resulted in minimal sedimentation of either polymer (Fig. 8B). Likewise, f-actin combined with microtubules resulted in little pelleting of either polymer (Fig. 8B). In contrast, f-actin combined with microtubules and MBPs resulted in pelleting of both microtubules and f-actin (Fig. 8B). Thus, microtubule-f-actin interaction is dependent on one or more MBPs.

\section{DISCUSSION}

The importance of microtubule-f-actin interactions for cytokinesis and directed cell locomotion has been apparent for many years. However, due to a number of technical problems (see Introduction), the nature of the interaction between microtubules and actomyosin has remained mysterious. The use of Xenopus egg lysates has allowed us to overcome 


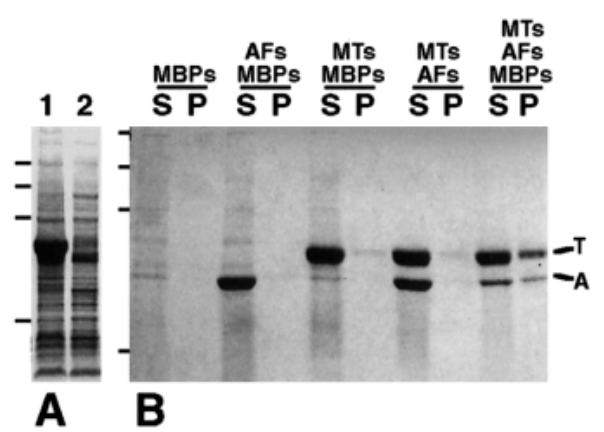

Fig. 8. F-actin associates with microtubules in the presence, but not the absence, of oocyte microtubule-binding proteins. (A) Coomassiestained gel showing pellet containing microtubules and microtubule binding proteins (lane 1) and the supernatant containing salt and ATP extracted microtubule binding proteins (lane 2). (B) Coomassiestained gel showing microtubule-binding protein (MBP)-dependent cosedimentation of actin filaments (AFs) and microtubules (MTs). Little pelleting of f-actin or microtubules occurs at low speeds when the two are incubated separately with microtubule binding proteins or together without microtubule binding proteins. However, when all three are incubated together, both microtubules and f-actin pellet at low speeds. Bars at the left of each gel indicate the molecular mass markers (from top: $200,130,80$, and $31 \mathrm{kDa}$ ). A indicates position of actin; $T$ indicates position of tubulin.

limitations imposed by classic fixation-based approaches. The results obtained here with Xenopus egg extracts provide, to the best of our knowledge, the first demonstration that microtubules associate with f-actin under conditions that approximate the situation in vivo.

The use of Xenopus egg lysates to study the cytoskeleton under conditions that mimic the in vivo state is certainly not novel (e.g. Verde et al., 1990; Belmont et al., 1990; Theriot et al., 1994; Ma et al., 1998; Moreau and Way, 1998), but since this is the first use of this system to study microtubule-f-actin interactions, it is important to address the possibility that the observed association is simply an artifact of nonspecific interactions between the two polymer systems that might occur as a result of the experimental design. The following findings indicate that the observed association of microtubules with actomyosin is strong, specific and biologically relevant: (1) Factin-microtubule association survives multiple (at least 15) washes; (2) F-actin associates in a predominantly lengthwise fashion with astral microtubules, rather than simply being intertwined with them as would be expected if the interactions were nonspecific; (3) Except in the cases of the f-actin arcs, which resemble f-actin comets nucleated from vesicles in Xenopus egg extracts (Ma et al., 1998; Moreau and Way, 1998), $\mathrm{f}$-actin associates with asters on the side distal to the direction of flow (i.e. at the plus ends of the microtubules), rather the proximal side, as would be expected if f-actin is simply trapped by the asters during the washing process; (4) Treatment of extracts with CD prior to addition to flow chambers results in straighter microtubules than controls, suggesting that the relevant interaction occurs before the process of preparation for viewing; (5) The position of the f-actin relative to the microtubules changes with increasing incubation time, an observation difficult to explain if the interaction is nonspecific, unless the change in distribution reflects differential accessibility of the f-actin probe; (6) F-actin associates with the microtubules of asters obtained by sedimentation through sucrose, indicating that the interaction is not an artifact of the flow chamber approach; (7) F-actin-microtubule association is also observed in the absence of asters, indicating that the interaction is not limited to large microtubule-containing structures; and (8) F-actin-microtubule interactions can be reconstituted with pure f-actin and pure microtubules in the presence, but not the absence of MBPs, indicating that the interaction is specific and mediated by one or more MBP intermediaries. Taken collectively, the results argue strongly that the observed association of $\mathrm{f}$-actin with microtubules in cell free lysates is not the result of nonspecific interactions between the two polymer systems, but instead represents a bona fide biochemical association.

A wealth of evidence indicates that perturbation of the microtubule cytoskeleton perturbs actomyosin-based cell motility. In addition to somehow directing cell locomotion and cytokinesis, microtubules negatively regulate actomyosinbased contraction of fibroblasts (Lyass et al., 1988; Danowski, 1989; Kolodney and Elson, 1995), and actomyosin-based cortical flow in Xenopus oocytes (Canman and Bement, 1997). How are the various lines of evidence of microtubuleactomyosin interactions to be interpreted? Two general models have been proposed. The first model, 'tensegrity', is essentially physical. Microtubules are viewed as providing extensive forces that passively resist contractile forces driven by actomyosin (Joshi et al., 1985; Dennerll et al., 1988; Ingber, 1993). The second 'regulatory' model, suggests that microtubules bind to factors that regulate the actomyosin cytoskeleton (Rappaport, 1986; Danowski, 1989; Rosanio and Swanson, 1996; Mikhailov and Gundersen, 1998), such that microtubules are able to modify the activity of the actomyosin cytoskeleton independently of strictly physical resistance to actomyosin-based contraction. The results of this study could be viewed as being consistent with either model, depending on one's perspective. From the 'tensegrity' standpoint, physical inhibition of contractile force by microtubules, would be increased by a direct or indirect linkage of the two systems. From the 'regulatory' standpoint, evidence for a linkage between microtubules and f-actin increases the probability that microtubules can modulate contractility by binding and sequestration of components of the actomyosin cytoskeleton. However, our results are also consistent with a third model, in which the microtubule cytoskeleton not only associates with the f-actin cytoskeleton, but also exerts force upon it. That is, the extensive lengthwise contact between the two systems could permit microtubule-based motors to transport f-actin. While the use of static specimens precludes us from making definitive statements about translocation, the observation that early asters have f-actin throughout the body of the aster while late asters have f-actin confined to outward edges of asters is certainly consistent with this notion.

In addition to addressing the modulation of actomyosin by microtubules, the results of this study are also relevant to the means by which actomyosin might regulate microtubules. The lengthwise association of f-actin with microtubules observed here is consistent with the recent proposal that f-actin may guide growing microtubules to focal contacts (Kaverina et al., 1998). Conversely, other reports have indicated that the cortical f-actin cytoskeleton exerts a negative influence on 
microtubules. For example, actin depolymerization results in increased microtubule polymerization, while f-actin-based contraction results in microtubule depolymerization (Dennerll et al., 1988). Similarly, depolymerization of f-actin results in engorgement of neuronal growth cones with microtubules (Forscher and Smith, 1988), while during growth cone-target contact, microtubules extend preferentially through areas of low f-actin density (Lin and Forscher, 1993). Further, recent analyses of microtubule dynamics in newt lung epithelia have shown that extending microtubules are swept away from the leading edge by actomyosin-dependent cortical flow and, in the process, are often bent or broken (Waterman-Storer and Salmon, 1997). The results of Waterman-Storer and Salmon (1997) using living cells, as well as our results showing that association with $\mathrm{f}$-actin promotes microtubule bending in vitro suggest that bent and kinky microtubules result from interaction with the actin cytoskeleton.

The precise molecules that link the microtubule and f-actin cytoskeletons have yet to be determined. We have shown that a MBP fraction is sufficient to permit microtubule-f-actin interaction. This finding is consistent with a number of reports from the literature. For example, neuronal microtubuleassociated proteins are capable of crosslinking microtubules and actin filaments in vitro (Griffith and Pollard, 1978, 1982), while smooth muscle caldesmon, an f-actin binding protein, binds to and bundles microtubules (Ishikawa et al., 1992). Candidates found in nonneuronal and nonmuscle cells include CLIP 170, a MBP that has recently been shown to associate with myosin-VI (Lantz and Miller, 1998), Mip-90, a MBP that colocalizes with both microtubules and f-actin (Gonzalez et al., 1998), plectin, a multifunctional cytoskeletal crosslinker that tethers intermediate filaments to both microtubules and the actomyosin cytoskeleton (e.g. Svitkina et al., 1996, and references therein), and coronin, which in yeast has been shown crosslink microtubules and f-actin, although it is not clear whether vertebrate coronin homologues bind microtubules (Goode et al., 1999). While we are not aware of any direct evidence that microtubule-based motors link f-actin to microtubules, it has recently been shown that kinesin can crosslink microtubules to vimentin filaments (Liao and Gundersen, 1998), and myosin- $\mathrm{V}$ has been reported to bind directly to kinesin (Huang et al., 1999) suggesting that motordependent linkage between different cytoskeletal systems is at least plausible.

Whether any of the above proteins serves to link microtubules and f-actin remains to be determined. However, the assays described in this report, coupled with protein fractionation approaches, should permit systematic analysis and eventual identification of proteins involved in microtubulef-actin crosslinking. Further, the Xenopus egg extract system should also prove useful for investigation of other, fundamental questions relating to cell motility. For example, the analysis of dynamic microtubule-f-actin interactions which is presently difficult in intact cells due to high levels of background actin signal should be more tractable in extracts. By combining such in vitro approaches with in vivo approaches, it is hoped that an integrated view of microtubule-f-actin interactions and their consequences will be achieved.

We are grateful to several of our colleagues for providing information, advice, and reagents: Peter Baas, Gary Borisy, Tom
Keating, Catherine Marler and Volodia Rodionov, University of Wisconsin, Madison. The approach employed in this study came out of preliminary experiments performed in the Physiology Course at the Marine Biological Laboratory (MBL), Woods Hole, MA. Support for this work was provided by grants from the National Science Foundation (MCB 9630860) and National Institutes of Health (GM52932-01A2) to W.M.B and a postdoctoral NSERC grant to C.A.M. The MBL Physiology Course is supported by the National Institutes of Health and the Howard Hughes Foundation; The Keck Neural Imaging Laboratory is supported by the National Institutes of Health.

\section{REFERENCES}

Allan, V. J. and Vale, R. D. (1991). Cell cycle control of microtubule-based membrane transport and tubule formation in vitro. J. Cell Biol. 113, 347359.

Belmont, L. D., Hyman, A. A., Sawin, K. E. and Mitchison, T. J. (1990). Real time visualization of cell cycle-dependent changes in microtubule dynamics in cytoplasmic extracts. Cell 62, 579-589.

Bement, W. M. and Capco, D. G. (1989). Activators of protein kinase C trigger cortical granule exocytosis, cortical contraction and cleavage furrow formation in Xenopus laevis oocytes and eggs. J. Cell Biol. 108, 885-892.

Canman, J. C. and Bement, W. M. (1997). Microtubules suppress actomyosin-based cortical flow in Xenopus oocytes. J. Cell Sci. 110, $1907-$ 1917.

Cooper, J. A. (1986). Effects of cytochalasin and phalloidin on actin. J. Cell Biol. 105, 1473-1478.

Danowski, B. A. (1989). Fibroblast contractility and actin organization are stimulated by microtubule inhibitors. J. Cell Sci. 93, 255-266.

Dennerll, T. J., Joshi, H. C., Steel, V. L., Buxbaum, R. E. and Heidemann, S. R. (1988). Tension and compression in the cytoskeleton of PC-12 neurites II: Quantitative measurements. J. Cell Biol. 107, 665-674.

Field, C., Li, R. and Oegema, K. (1999). Cytokinesis in eukaryotes: a mechanistic comparison. Curr. Opin. Cell Biol. 11, 68-80.

Fishkind, D. J. and Wang, Y.-L. (1995). New horizons for cytokinesis. Curr. Opin. Cell Biol. 7, 23-23-31.

Forscher, P. and Smith, S. J. (1988). Actions of cytochalasins on the organization of actin filaments and microtubules in a neuronal growth cone. J. Cell Biol. 107, 1505-1516.

Fujiwara, K. and Pollard, T. D. (1976). Fluorescent antibody localization of myosin in the cytoplasm, cleavage furrow and mitotic spindle of human cells. J. Cell Biol. 71, 848-875.

Gail, M. H. and Boone, C. W. (1971a). Effect of colcemid on fibroblast motility. Exp. Cell Res. 65, 221-227.

Gail, M. H. and Boone, C. W. (1971b). Cytochalasin effects on BALB/3T3 fibroblasts: dose-dependent, reversible alteration of motility and cytoplasmic cleavage. Exp. Cell Res. 68, 226-228.

Gavin, R. H. (1997). Microtubule-microfilament synergy in the cytoskeleton. Int. Rev. Cytol. 173, 207-242.

Goldman, R. D. (1971). The role of three cytoplasmic fibers in BHK-21 cell motility. I. Microtubules and the effects of colchicine. J. Cell Biol. 51, 752762

Gonzalez, M., Cambiazo, V. and Maccioni, R. B. (1998). The interaction of Mip-90 with microtubules and actin filaments in human fibroblasts. Exp. Cell Res. 239, 243-253.

Goode, B. L., Wong, J. J., Butty, A.-C., Peter, M., McCormack, A. L,. Yates, J. R., Drubin, D. G. and Barnes, G. (1999). Coronin promotes the rapid assembly and cross-linking of actin filaments and may link the actin and microtubule cytoskeletons in yeast. J. Cell Biol. 144, 83-98.

Griffith, L. M. and Pollard, T. D. (1978). Evidence for actin filamentmicrotubule interaction mediated by microtubule associated proteins. J. Cell Biol. 78, 958-965.

Griffith, L. M. and Pollard, T. D. (1982). The association of actin filaments with microtubules and microtubule-associated proteins. J. Biol. Chem. 257, 9143-9151.

Hamaguchi, Y. (1975). Microinjection of colchicine into sea urchin eggs. Dev. Growth Differ. 17, 111-117.

Hiramoto, Y. (1956). Cell division without mitotic apparatus in sea urchin eggs. Exp. Cell Res. 11, 630-636. 
Hiramoto, Y. (1971). Analysis of cleavage stimulus by means of micromanipulation of sea urchin eggs. Exp. Cell Res. 68, 291-298.

Howard, T. H. and Oresajo, C. O. (1985). A method for quantifying f-actin in chemotactic peptide activated neutrophils: study of the effect of tBOC peptide. Cell Motil. 5, 545-57.

Huang, J.-D., Brady, S. T., Richards, B. W., Stenoien, D., Resau, J. H., Copeland, N. G. and Jenkins, N. A. (1999). Direct interaction of microtubule- and actin-based transport motors. Nature 397, 267-270.

Ingber, D. E. (1993). Cellular tensegrity: defining new rules of biological design that govern the cytoskeleton. J. Cell Sci. 104, 613-627.

Ishikawa, R., Kagemi, O., Hayashi, C. and Kohama, K. (1992). Characterization of smooth muscle caldesmon as a microtubule-associated protein. Cell Motil. Cytoskel. 23, 244-251.

Joshi, H. C., Chu, D., Buxbaum, R. E. and Heidemann, S. R. (1985). Tension and compression in the cytoskeleton of PC12 neurites. J. Cell Biol. 101, 697-705.

Kaverina, I., Rottner, K. and Small, J. V. (1998). Targeting capture and stabilization of microtubules at early focal adhesions. J. Cell Biol. 142, 181190.

Kolodney, M. S. and Elson, E. L. (1995). Contraction due to microtubule disruption is associated with increased phosphorylation of myosin regulatory light chain. Proc. Nat. Acad. Sci. USA 92, 10252-10256.

Lantz, V. A. and Miller, K. G. (1998). A class VI unconventional myosin is associated with a homologue of a microtubule-binding protein, cytoplasmic linker protein-170, in neurons and at the posterior pole of Drosophila embryos. J. Cell Biol. 140, 897-910.

Leno, G. H. and Laskey, R. A. (1991). DNA replication in cell-free extracts from Xenopus laevis. Meth. Cell Biol. 36, 561-579.

Liao, G. and Gundersen, G. G. (1988). Kinesin is a candidate for crossbridging microtubules and intermediate filaments. J. Biol. Chem. 273, $9797-$ 9803 .

Lin, C.-H. and Forscher, P. (1993). Cytoskeletal remodeling during growth cone-target interactions. J. Cell Biol. 121, 1369-1383.

Lyass, L. A., Bershadsky, A. D., Vasiliev, J. M. and Gelfand, I. M. (1988). Microtubule-dependent effect of phorbol ester on the contractility of cytoskeleton of cultured fibroblasts. Proc. Nat. Acad. Sci. USA 85, 95389541.

Ma, L., Cantley, L. C., Janmey, P. A. and Kirschner, M. W. (1998). Corequirement of specific phosphoinositides and small GTP-binding protein cdc42 in inducing actin assembly in egg extracts. J. Cell Biol. 140, 11251136

Mabuchi, I. and Okuno, M. (1977). The effect of myosin antibody on the division of starfish blastomeres. J. Cell Biol. 74, 251-263.

McBeath, E. and Fujiwara, K. (1984). Improved fixation for immunofluorescence microscopy using light-activated 1,3,5-triazido-2,4,6trinitrobenzene (TTB). J. Cell Biol. 99, 2061-2073.

Mikhailov, A. and Gundersen, G. G. (1998). Relationship between microtubule dynamics and lamellipodium formation revealed by direct imaging of microtubules in cells treated with nocodazole or taxol. Cell Motil. Cytoskel. 41, 325-340

Moreau, V. and Way, M. (1998). Cdc42 is required for membrane dependent actin polymerization in vitro. FEBS Letts. 427, 353-356.
Murray, A. W. (1991). Cell cycle extracts. Meth. Cell Biol. 36, 581-605.

Newmeyer, D. D. and K. L. Wison. (1991). Nuclear import and assembly reactions. Meth. Cell Biol. 36, 607-634.

Oegema, K. and Mitchison, T. J. (1997). Rappaport rules: cleavage furrow induction in animal cells. Proc. Nat. Acad Sci. USA 94, 4817-4820.

Rappaport, R. (1961). Experiments concerning the cleavage stimulus in sand dollar eggs. J. Exp. Zool. 148, 81-91.

Rappaport, R. (1986). Establishment of the mechanisms of cytokinesis in animal cells. Int. Rev. Cytol. 105, 245-281.

Rickard, J. E. and Kreis, T. E. (1990. Identification of a novel nucleotidesensitive microtubule binding protein in HeLa Cells. J. Cell Biol. 110, $1623-$ 1633.

Rosanio, G. R. and Swanson, J. A. (1996). Microtubules can modulate pseudopod activity from a distance inside macrophages. Cell Motil. Cytoskel. 34, 230-245.

Schroeder, T. E. (1973). Actin in dividing cells: Contractile ring filaments bind heavy meromyosin. Proc. Nat. Acad. Sci. USA 70, 1688.

Stearns, T. and Kirschner, M. (1994). In vitro reconstitution of centrosome assembly and function: The central role of $\gamma$-tubulin. Cell $\mathbf{7 6}$ 623-637.

Svitkina, T. M., Verkhovsky, A. B. and Borisy, G. G. (1996). Plectin sidearms mediate interaction of intermediate filaments with microtubules and other components of the cytoskeleton. J. Cell Biol. 135, 991-1007.

Theriot, J. A., Rosenblatt, J., Portnoy, D. A., Goldschmidt-Clermont, P. J. and Mitchison, T. J. (1994). Involvement of profilin in the actin based motility of $L$. monocytogenes in cells and cell-free extracts. Cell 76, 505517

Vale, R. D., Reese, T. S. and Sheetz, M. P. (1985). Identification of a novel force-generating protein, kinesin, involved in microtubule-based motility. Cell 42, 39-50.

Vasiliev, J. M., Gelfand, I. M., Domnina, L. V., Ivanova, O. Y., Komm, S. G. and Olshevskaya, L. V. (1970). Effect of colcemid on the locomotory behavior of fibroblasts. J. Embryol. Exp. Morph. 24, 625-640.

Vasiliev, J. M. (1991). Polarization of pseudopodial activities: cytoskeletal mechanisms. J. Cell Sci. 98, 1-4.

Verde, F., Labbe, J., Doree, M. and Karsenti, E. (1990). Regulation of microtubule dynamics by cdc2 protein kinase in cell free extracts of Xenopus eggs. Nature 343, 233-243.

Vielkind, U. and Swierenga, S. H. (1989). A simple fixation procedure for immunofluorescence detection of different cytoskeletal components within the same cell. Histochemistry 91, 81-88.

Waterman-Storer, C. M. and Salmon, E. D. (1997). Actomyosin-based retrograde flow of microtubules in the lamella of migrating epithelial cells influences microtubule dynamic instability and turnover and is associated with microtubule breakage and treadmilling. J. Cell Biol. 139, 417-434.

Waterman-Storer, C. M. and Salmon, E. D. (1999). Positive feedback interactions between microtubule and actin dynamics during cell motility. Curr. Opin. Cell Biol. 11, 61-67.

Zigmond, S. H. and Hirsch, J. G. (1972). Effects of cytochalasin B on polymorphonuclear leucocyte locomotion, phagocytosis and glycolysis. Exp. Cell Res. 73, 383-393. 